\title{
THE COEFFICIENT OF EXPANSION OF NICKEL AT ITS CRITICAL TEMPERATURE.
}

By H. M. Randall.

RON at its critical temperature shows other remarkable physical changes, such as the phenomena of recalescence, a fall in the temperature coefficient of electrical resistance, and a marked change in its thermo-electric properties. A decided change in the nature of iron seems to occur at this temperature.

Nickel has a similar critical temperature, but its loss of magnetization at this temperature is much more gradual than in the case of iron, and the other physical changes which might be expected to accompany this loss are not so marked as in the case of iron. Hopkinson ${ }^{1}$ was not able to find any trace of the phenomenon of recalescence as nickel changes from the non-magnetic to the magnetic state. Recently it has been shown ${ }^{2}$ that there is a change in the thermo-electromotive force and in the temperature coefficient of electrical resistance in this neighborhood, and Holborn and Day ${ }^{3}$ have found that there is an anomalous change in the coefficient of thermal expansion at the critical temperature.

It was thought that the method embodied in the use of the Pulfrich interferometer would be well adapted to the determination of the exact nature of any change in the temperature coefficient of expansion at this temperature. The arrangement of apparatus employed was substantially that used by the author in the investigation of the coefficient of expansion of quartz. ${ }^{4}$ The nickel used was in the form of a cylinder of pure nickel furnished by Zeiss, Jena, $2.2 \mathrm{~cm}$. in diameter and $9.690 \mathrm{~mm}$. in height. Its upper surface was plane polished, and its lower surface, parallel to the upper, was ground away with the exception of three equidistant feet. This cylinder was mounted on the quartz bed-plate within the

1 Proc. Roy. Soc., Vol. XLIV., I888, p. 317.

${ }^{2}$ Campbell, Elect. Rev., 48, June 14, I901, p. Ior4-1015; also, Harrison, Nature, October 31, 190I ; Phil. Mag., February, 1902.

3 Ann. der Phys., 309, p. 104.

4 Phys. Rev., January, 1905. 
[VOL. XX.

quartz ring, one of its feet having been ground away till its upper surface had the proper inclination to the lower surface of the cover plate to give interference fringes of the desired width. As the air layer had a thickness of approximately one tenth of a millimeter, the red and blue fringe systems of hydrogen vapor were very bright and they were used together with the green mercury system. At the higher temperatures the polished surface of the nickel became colored a deep blue, the result being that the red was so poorly reflected that its fringe system could no longer be used.

If $\alpha$ be the coefficient of expansion of nickel to be determined, and $\beta$ be the known coefficient of expansion of the quartz ring, while $N$ and $L$ represent the lengths of the nickel cylinder and quartz ring respectively, then $d$, the change of thickness of the air layer due to a change in temperature, $t_{2}-t_{1}$, may be represented by the following expression in which $f$ equals the number of displaced fringes, and $\lambda$ the wave-length of the spectral color used.

from which

$$
\left.d=f \lambda / 2=N t_{2}-t_{1}\right)-L_{i} \beta\left(t_{2}-t_{1}\right)
$$

$$
\mu=\frac{f_{2}^{\lambda}}{N\left(t_{2}-t_{1}\right)}+\frac{L}{N}{ }^{\beta}
$$

for the mean temperature $\left(t_{2}+t_{1}\right) / 2$.

The results obtained are given in the following tables. Table I. and Curve I., represent a series of determinations extending from room temperature to $200^{\circ} \mathrm{C}$. At this point the quartz apparatus seemed to have become disarranged and the apparatus was dismounted. Some three months later the work was again undertaken, the mean temperature for the first determination being in the neighborhood of $200^{\circ} \mathrm{C}$. A series of results obtained at this point with alternate rising and falling temperature showed that the coefficient of expansion of the nickel was'very markedly lowered by each rise and subsequent fall of temperature. To get a series of results at all regular it became necessary to have all the temperature changes take place in the same direction. Accordingly, after allowing the apparatus to stand a week at room temperatures, a second series, Table II. and Curve II., was obtained, in which the temperature intervals were all formed by increasing the temperature. 
No. 2.] THE COEFFICIENT OF EXPANSION OF NICKEL.

TABLE I.

\begin{tabular}{|c|c|c|c|c|c|c|c|}
\hline$t_{1}$ & $t_{2}$ & $\begin{array}{c}t_{1}+t_{2} \\
2\end{array}$ & $a \times 10^{8}$ & $t_{1}$ & $t_{2}$ & $\begin{array}{c}t_{1}+t_{2} \\
2\end{array}$ & $a \times 10^{8}$ \\
\hline 20.0 & 45.5 & 32.8 & 1326 & 76.7 & 149.9 & 113.3 & 1443 \\
\hline 45.5 & 66.2 & 55.9 & 1356 & 103.4 & 149.9 & 126.6 & 1456 \\
\hline 53.6 & 76.7 & 65.2 & 1356 & 150.2 & 181.9 & 166.1 & 1513 \\
\hline 76.7 & 103.4 & 90.1 & 1399 & 181.9 & 214.2 & 198.1 & 1581 \\
\hline 76.7 & 150.2 & 113.5 & 1431 & & & & \\
\hline
\end{tabular}

TABLE II.

\begin{tabular}{r|c|c|c||c|c|c|c}
\hline \hline \multicolumn{1}{c|}{$t_{1}$} & \multicolumn{1}{|c|}{$t_{2}$} & $\begin{array}{c}t_{1}+t_{2} \\
\mathbf{2}\end{array}$ & $a \times \mathrm{xO}^{8}$ & $t_{1}$ & $t_{2}$ & $\begin{array}{c}t_{1}+t_{2} \\
2\end{array}$ & $\alpha \times$ I0 $^{*}$ \\
\hline 63.6 & 105.4 & 84.5 & 1362 & 274.2 & 293.0 & 283.6 & 1637 \\
105.4 & 137.5 & 121.4 & 1405 & 283.1 & 303.9 & 293.5 & 1638 \\
137.5 & 157.5 & 147.5 & 1442 & 293.0 & 315.4 & 304.2 & 1652 \\
157.5 & 178.7 & 168.1 & 1471 & 303.9 & 328.4 & 316.1 & 1649 \\
178.7 & 193.8 & 186.3 & 1491 & 315.4 & 337.6 & 326.5 & 1637 \\
193.8 & 214.1 & 203.4 & 1517 & 328.4 & 346.3 & 337.3 & 1643 \\
215.2 & 239.2 & 227.2 & 1577 & 337.6 & 357.5 & 347.6 & 1635 \\
239.2 & 260.0 & 249.6 & 1611 & 346.3 & 368.1 & 357.2 & 1645 \\
260.0 & 274.2 & 267.1 & 1630 & 357.5 & 378.8 & 368.1 & 1640 \\
260.0 & 283.1 & 271.6 & 1630 & 378.8 & 404.9 & 391.8 & 1664 \\
\hline
\end{tabular}

As the table and plot show, in the region included between $280^{\circ}$ and $370^{\circ}$, which is generally recognized as the region of the critical

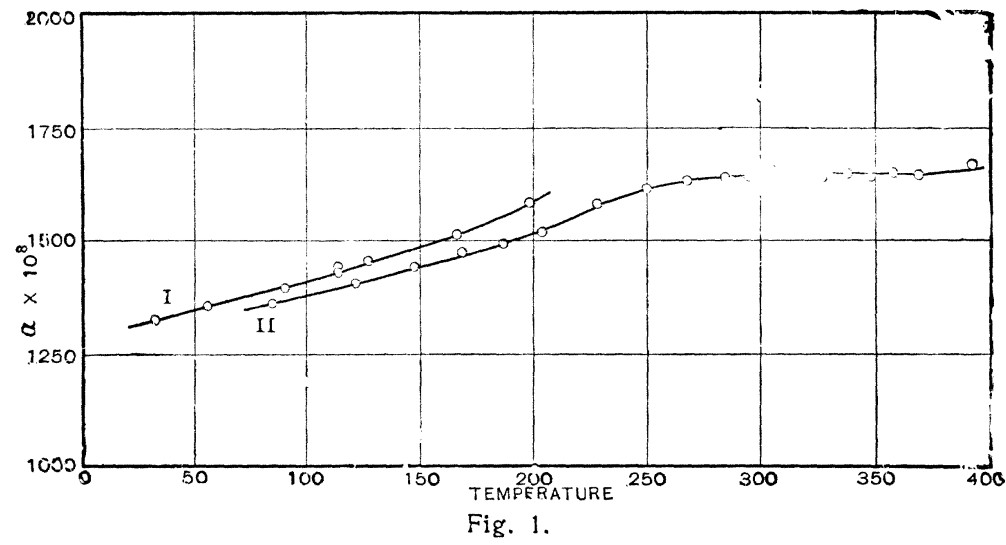

temperature, the individual values of the coefficient of expansion do not deviate from a constant value by more than would be expected from experimental error. The result obtained at $392^{\circ} \mathrm{C}$. would seem to indicate that the coefficient was again increasing with a rise 
in temperature. This determination cannot be given the weight of the preceding ones, however, as the nickel began to expand so unequally after passing $350^{\circ} \mathrm{C}$. that the interference bands became distorted, and this distortion became so great after passing $400^{\circ} \mathrm{C}$. that all further progress was stopped. Upon cooling the polished surface did not again become plane.

Harrison ${ }^{1}$ has recently found, working with nickel wire, an anomalous expansion between $365^{\circ} \mathrm{C}$. and $370^{\circ} \mathrm{C}$. amounting to approximately $0.04 \mathrm{~mm}$. on a length of $10 \mathrm{~cm}$. An equivalent expansion of the nickel cylinder would result in a displacement of approximately 10 bands. Between $300^{\circ} \mathrm{C}$. and $400^{\circ} \mathrm{C}$. the number of displaccd bands corresponding to a temperature interval of $20^{\circ}$, determined by direct counting and also by computation after the method of Abbé, did not at any time exceed three. The relative expansion is necessarily small, as the nickel cylinder and the quartz ring have nearly the same length and, in this temperature region, their coefficients of expansion do not differ greatly. An anomalous expansion represented by the passage of IO bands could therefore hardly have escaped notice. It must be concluded then, that the nickel cylinder used in this investigation does not show, within the temperature limits employed, an anomalous expansion of the magnitude of that found by Harrison with nickel wire, and that, if such a phenomenon occurs, it must be at a temperature above $400^{\circ} \mathrm{C}$.

As has been shown by Holborn and Day, ${ }^{2}$ to get definite results with nickel it is necessary to first heat it to the maximum temperature to be employed and to allow it to cool before making measurements. The nickel cylinder, not having been thus treated before being polished, neither set of results can justly be considered as representing the coefficient of expansion of nickel, and it has not seemed worth while, therefore, to express them by means of equations. It is expected in the near future to anneal thoroughly the nickel cylinder, by repeated heatings, and repolish its surface, after which a careful determination of its coefficient of expansion will again be undertaken.

Physical Laboratory, University of Michigan, ANn Arbor, September 9, 1904.

'Phil. Mag., June, 1904.

${ }^{2}$ Loc. cit. 